\title{
Eye Torsion Associated with Disparity-Induced Vertical Vergence in Humans
}

\author{
L. J. VAN RIJN, ${ }^{* \dagger}$ H. COLLEWIJN* \\ Received 27 October 1993; in revised form 27 December 1993
}

\begin{abstract}
Recently, Enright described an unexpected association between disparity-induced vertical vergence and cycloversion (conjugate eye torsion) [Enright (1992) Vision Research, 415, 279]. The present experiments were performed to verify these findings and investigate the nature of this association. We presented subjects with a dichoptic image of concentric circles in which a step in vertical disparity of 1 deg was introduced. After 4 sec the disparity was eliminated. Eye movements were measured with scleral coils. We confirmed Enright's findings in that a left-over right vertical vergence was associated with levo-cycloversion (upper poles towards left shoulder) and vice versa. The size of the cycloversion and the vertical vergence were in the same range. In addition we found that part of the cycloversion response was in the form of a torsional nystagmus and that the relative contribution of the left and right eyes was independent of the horizontal gaze angle. These additional findings are in conflict with the hypothesis, offered by Enright, that the association is caused by a bilateral activity of the superior oblique muscles.
\end{abstract}

Eye movements Cycloversion Vertical vergence Human coils

\section{INTRODUCTION}

Binocular, single vision requires optimal correspondence between the images on the retinas of the two eyes. Such correspondence is achieved by horizontal and vertical alignment of the two lines of sight, such that they intersect in a single binocular fixation point, as well as by correct orientation of each eye around the axis formed by its line of sight. The horizontal distance between the eyes, combined with the variable distance of objects, necessitates a large degree of freedom in the horizontal angle between the eyes, which is manifest as a large range of horizontal vergence eye movements. In natural conditions, horizontal convergence is associated with accommodation and pupillary constriction (the near triad); the functional significance of this association is obvious.

Recently, we (Van Rijn \& Van den Berg, 1993) described the association of cyclovergence with horizontal vergence and elevation (vertical gaze angle), and argued that this linkage, formally described as a threedimensional extension of Listing's law, serves to optimize torsional correspondence. Accordingly, only limited independent control of cyclovergence by cy-

\footnotetext{
*Department of Physiology I, Faculty of Medicine, Erasmus University Rotterdam, P.O. Box 1738, 3000 DR Rotterdam, The Netherlands.

tTo whom all correspondence should be addressed.

$\$$ We define all eye rotations with respect to the subject. See Methods.
}

clodisparity has been demonstrated (Kertesz, 1983; Howard \& Zacher, 1991; Van Rijn, Van der Steen \& Collewijn, 1992).

In a similar vein, the vertical alignment of the eyes is usually constrained by the need to keep both lines of sight within a single plane of regard. Accordingly, independent control of vertical gaze angles (vertical vergence) is limited (Kertesz, 1981). The limited range and dynamics of vertical vergence and cyclovergence (as far as the latter is independent of horizontal vergence and elevation) suggest that they are mainly involved in the slow reduction of vertical and cyclo-disparities, resulting from imperfections in binocular motor control, rather than in a moment-to-moment control of binocular gaze.

Recently, Enright (1992) described an association between two movement directions which seems less readily interpretable in functional terms such as sketched above. He reported that disparity-induced vertical vergence was associated with conjugate eye torsion, i.e. cycloversion. Left-over-right ( $L / R)$ vertical vergence (i.e. elevation of the left eye above the right eye) was associated with counter-clockwise torsion in both eyes, i.e. rotation of both upper poles towards the left shoulder; $;$ right-over-left vertical vergence $(R / L)$ with clockwise torsion. Enright also found vertical vergence to be combined with slight lateral translations of the eyes. Therefore, he hypothesized that this torsion was caused by the fact that vertical vergence was mediated predominantly by the superior oblique muscles. This would 
indicate a mechanical constraint rather than a functional significance as the basis for this linkage.

Enright obtained his results using video-oculography, which offers a very limited temporal resolution. The first purpose of the present study was to reproduce these unanticipated results with scleral coils. $\Lambda$ fter we confirmed Enright's findings we tried, as a second goal, to gain insight in the mechanism of this phenomenon. For this purpose, we investigated the dynamic relation between the vertical vergence anc cycloversion. The superior-oblique hypothesis of Enright predicts this relation to be very tight. Secondly, according to this hypothesis one should expect that an abducted eye displays more torsion than an adducted eye because in the latter, the primary action of the superior oblique muscle is depression and not torsion. We tested this prediction by measuring the gaze dependency of the contribution of the left and right eyes to the cycloversion response.

The results indicate that the association between vertical vergence and cycloversion is indeed present, at least, this was the case in four out of our six subjects. However, in some of these subjects the torsional responses were partly in the form of a cycloversional nystagmus. The contribution of the left and right eyes was independent of horizontal gaze angle. These findings are in conflict with the superior-oblique hypothesis of Enright.

\section{METHODS}

\section{Subjects}

Six human subjects (three females and three males) participated in these experiments after giving informed consent. Their age ranged from 26 to $44 \mathrm{yr}$. One of them (subject 5) wore spectacles (S $-2.5 \mathrm{D}$, ODS). All had a visual acuity of at least 20/20 in each eye (with spectacle correction) and a stereo-acuity of at least $60 \mathrm{sec}$ arc, as measured with the TNO fly-test (Medical Workshop, Groningen). Phorias were determined in horizontal and vertical directions using a Maddox cross. The test was performed with the subject at $145 \mathrm{~cm}$ distance from the cross since this was the distance of the visual stimulus during the experiment. Horizontal and vertical phorias were small: vertical phorias were less than $0.6 \mathrm{deg}$ (see Table 1); horizontal phorias ranged from $1.32 \mathrm{deg}$ eso to 0.06 deg exo.

\section{Recording of eye positions}

Eye positions were measured with scleral induction coils of the combination type (Ferman, Collewijn, Jansen \& Van den Berg, 1987) according to the technique of Robinson (1963). Eye position signals were amplified, low-pass filtered, digitized at $125 \mathrm{~Hz}$ and stored on disk by a PDP 11/73 minicomputer, for off-line analysis. Prior to each experiment, all channels were calibrated with the coils mounted on a protractor device, near the centre of the magnetic field. During the first measurement of each session subjects fixated a small dot that was straight ahead with respect to the coil system The steady offset values from this measurement represented coil misalignment. Correction for this misalignment was performed by a matrix transformation (Ferman et al.. 1987) using these steady offset values. [We neglected the small adduction of each visual axis (about $1.3 \mathrm{deg}$ ) due to the finite distance $(145 \mathrm{~cm})$ of the target.] All eye rotations were expressed in Fick coordinates (see e.g. Robinson, 1963; Carpenter, 1988). Therefore, torsion is expressed as rotation about the line of sight. All eye deviations are defined with respect to the subject. Hence, levo-cycloversion means that the upper poles of the eyes rotate towards the subject's left side

\section{Protocol and visual stimulus}

Subjects were seated with their eyes near the centre of the magnetic field of the eye position measurement system. Their heads were supported by chin and forehead rests. The visual stimulus was backprojected on a translucent screen at $145 \mathrm{~cm}$ distance in front of the subject. This stimulus, subtending $43 \mathrm{deg}$ of visual angle, consisted of five concentric circular rings, each about 2 deg wide (see Fig. 1). It was presented to the subjects dichoptically; two images were presented by separate slide projectors with red and green filters. Filters of the same material were mounted on a pair of spectacles worn by the subject. The position of the images of the left and right eyes could be controlled by mirrors, attached to scanners (General Scanning. Watertown, Mass., Model MG 350D). This stimulus configuration was designed to enable control of vertical disparity without providing any reference for cyc torsion.

Each experiment consisted of 16 trials (plus calibrations). Each trial lasted $12 \mathrm{sec}$. At the start of a trial, no disparity was present; after $4 \mathrm{sec}$ a vertical disparity was introduced, either left-over-right $(\mathrm{L} / \mathrm{R})$ or right-over-left

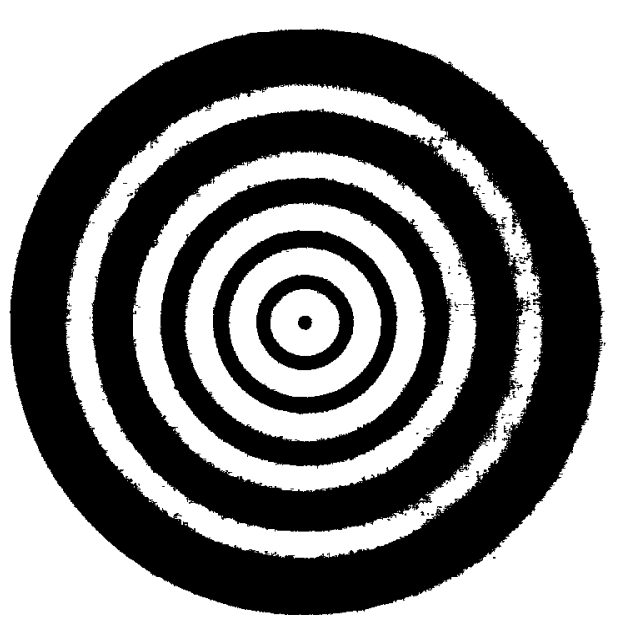

FIGURE 1. The stimulus pattern used in these experiments. Concentric circles were used so as to be able to present vertical disparities and leave torsion free. The width of each white annulus was about $2 \mathrm{deg}$. The width of the black annuli increased in the visual periphery, to compensate for decreased visual acuity. The outer border of the peripheral black circle could not be perceived in the dark room; the stimulus size ( $43 \mathrm{deg}$ of visual angle) is measured as the diameter of the outer border of the outer white annulus. 
(R/L). Again $4 \mathrm{sec}$ later this disparity was eliminated. Introduction and elimination of the disparities were step-like. The size of the disparity was $1 \mathrm{deg}$. This appears to be about the maximum vertical disparity that can be fused when presented in a step (Perlmutter \& Kertesz, 1978). The presentation of the disparity was symmetric in the sense that the displacements of the images to the left and right eyes were equal but opposite in magnitude $( \pm 0.5 \mathrm{deg})$. The duration of the disparity presentation was limited to $4 \mathrm{sec}$ in order to restrict the contamination of cycloversion by spontaneous variation (Van Rijn, Van der Steen \& Collewijn, 1994). Subjects were instructed to fixate the centre of the visual stimulus, but, if possible, to appreciate the stimulus as a whole and to achieve a single, fused image. Subjects were asked to abstain from blinking during trials. Throughout the experiment, the room was darkened.

An additional experiment in subject 6 was performed using the same protocol, except that the head was fixated by a biteboard. An additional experiment in subject 2 consisted of 36 trials (plus calibrations). The protocol was identical except that the stimulus was presented either straight ahead or $20 \mathrm{deg}$ left or right from the straight ahead.

\section{Data analysis}

Prior to analysis, all data were inspected for the occurrence of blinks; $13 \%$ of the trials were excluded on this ground.

After correction for coil misalignment (see above), vertical vergence, cyclovergence and cycloversion were calculated for each data sample. Vergence was calculated as left eye position minus right eye position (hence cyclovergence $=$ left eye torsion - right eye torsion) and version as the mean of the position of the left and right eye. Data shown in Fig. 2 are typical examples of recordings for all subjects obtained in this way.

Saccades were removed from the cycloversion traces by a computer routine. A saccade was defined as any period during which torsional eye velocity exceeded $3 \mathrm{deg} / \mathrm{sec}$. This criterium proved to remove the majority of small torsional saccades without affecting slow torsional eye movements. Cycloversion with saccades removed will be denoted as "smooth cycloversion".

For the purpose of averaging, sample numbers were reduced by a factor 12 , i.e. every 12 consecutive samples were replaced by one sample that represented the mean of these 12 samples. Subsequently, samples were averaged across the trials with a similar disparity direction in one session. Averaged responses are shown in Fig. 3.

\section{Statistical analysis}

Exponential curve-fits of vertical vergence responses were performed using the program Slide-Write 5.0 (Advanced Graphics Software Inc., Carlsbad, Calif.). In this program, curves are fitted by an iterative process, minimizing the value of $\chi^{2}$. Curve-fit parameters were tested for differences with the MANOVA routine from SPSSPC statistical package.

\section{RESULTS}

\section{Vertical vergence}

Vertical eye movements were recorded in five of our six subjects. (Due to technical problems, in subject 4 only torsional movements were recorded). All of them displayed clear vertical vergence responses to the vertical disparity-stimulus in either direction $(\mathrm{L} / \mathrm{R}$ or $\mathrm{R} / \mathrm{L})$. Examples of individual trials, for all subjects, are shown in Fig. 2. Averaged responses are shown in Fig. 3.

We computed the gain and time constant of the responses by fitting to these average curves an exponential curve of the type $\left[a_{0} *(1-\exp (-t / \tau))\right]$ for the response to the introduction of the disparity and $\left[a_{0} * \exp (-t / \tau)\right]$ for the response to its elimination. In these fits, $t$ is the elapsed time after the disparity step, $a_{0}$ is the asymptote of the response and $\tau$ is the time constant. In general the curve fit quality was good: $r^{2}$ was $0.984 \pm 0.021$ (mean \pm SD of all 20 fits). $a_{0}$ was $0.55 \pm 0.11$ (mean $\pm \mathrm{SD}$ ) and not significantly different between disparity introduction and elimination or between positive and negative disparities. Since the disparity step was $1 \mathrm{deg}$, this value of 0.55 equals the gain of the vertical vergence movement. $\tau$ was significantly shorter for disparity elimination as compared to introduction: $0.60 \pm 0.12 \mathrm{sec}$ as compared to $0.94 \pm 0.48 \mathrm{sec}$ (ANOVA: $P=0.030$ ). Time constants for positive and negative disparities were not significantly different.

\section{Cycloversion}

Figures 2 and 3, lower tracings, show the "raw" and "smooth" cycloversion responses, prior to, during and after the elimination of the vertical disparity. Figure 2 shows individual responses, Fig. 3 depicts averaged responses.

Spontaneous cycloversion. Prior to the introduction of the vertical disparity, subjects 2 and 4 showed a systematic drift of spontaneous cycloversion. This is best appreciated from the averaged smooth cycloversion tracings (Fig. 3). Both in subjects 2 and 4 this drift was in dextro-cycloversional direction.

Disparity introduction. The introduction of a vertical disparity was associated with vertical vergence and, in four of our six subjects (1-4), with conjugate eye torsion or cycloversion. $\mathrm{L} / \mathrm{R}$ vertical disparity was associated with levo-cycloversion; $R / L$ vertical disparity with dextro-cycloversion. A variable part of the cycloversion was in the form of a cycloversional nystagmus (with the slow phase in the indicated direction) rather than a tonic response. The magnitude of this nystagmoid part can be deduced from the difference between the "raw" and cumulative "smooth" cycloversion tracings in Fig. 3. In subjects 1 and 4 the response was almost exclusively tonic; in subjects 2 and 3 the responses were roughly half tonic and half nystagmoid. The averaged responses, shown in Fig. 3, demonstrate, in addition to the effects shown in Fig. 2, that cycloversion responses were exponential or linear, depending on the subject and the disparity direction. Because of this variability, curve fits were not performed on cycloversion data. Subject 5 


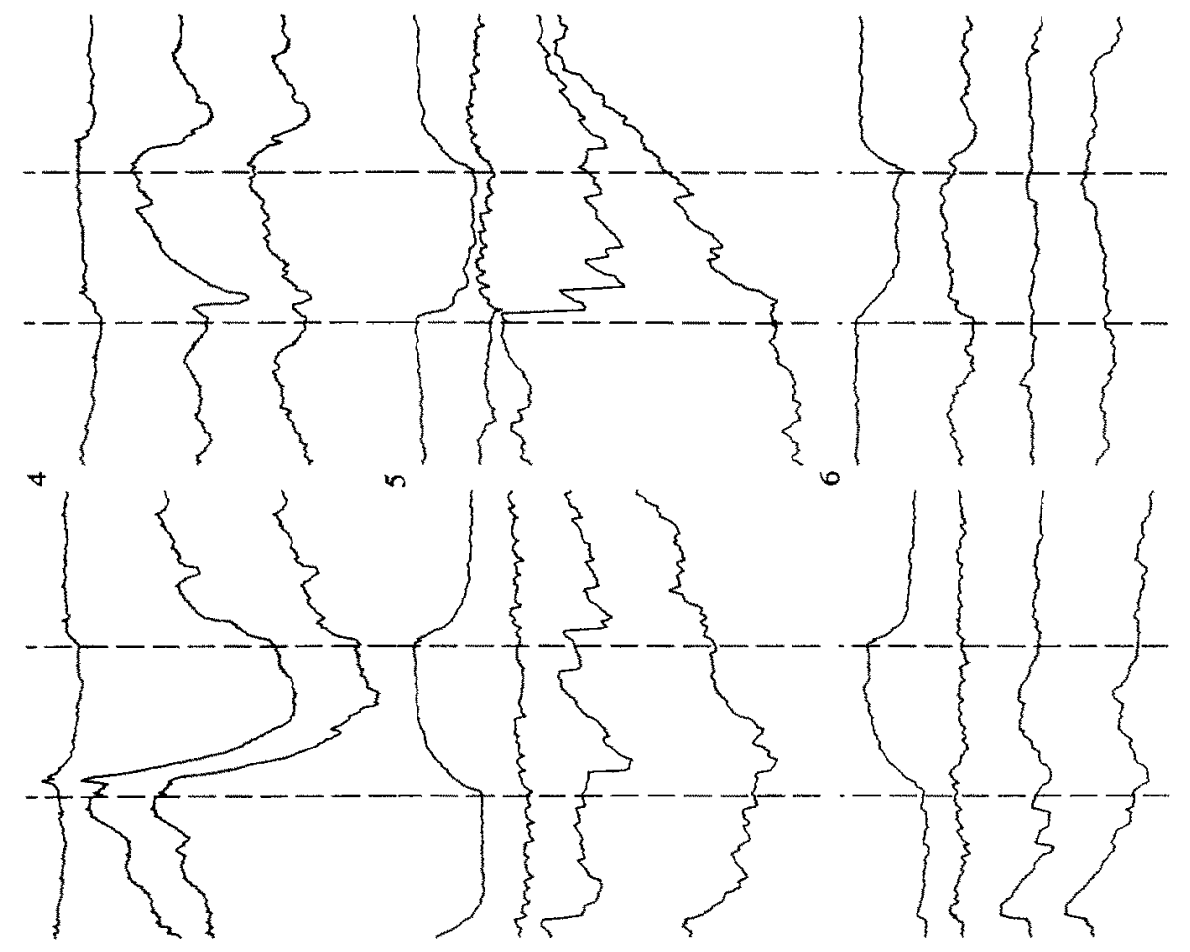

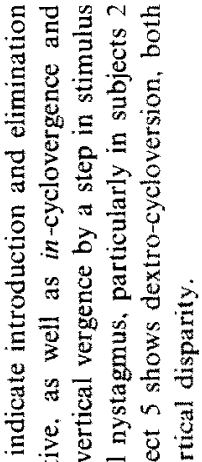

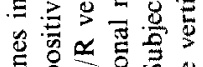

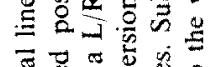

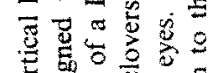

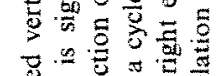

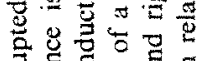

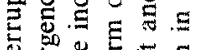

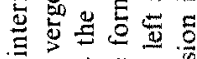

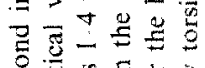

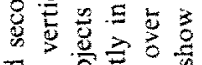

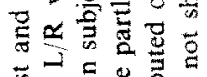

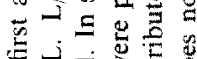

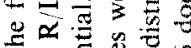
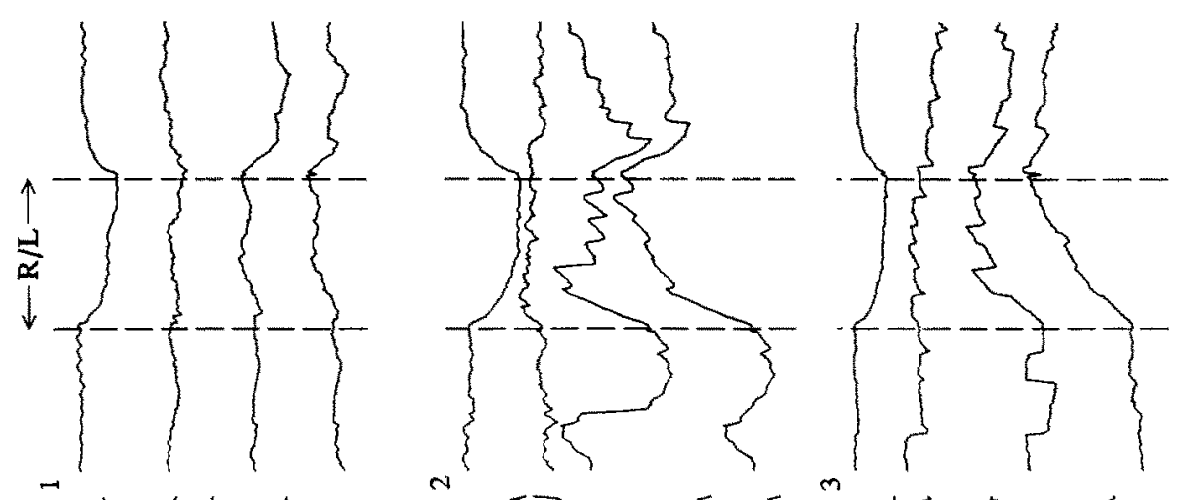

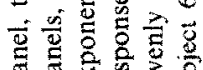

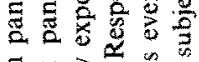

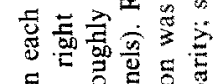

玉

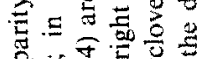

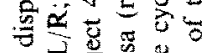

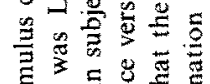

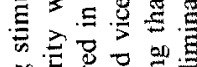
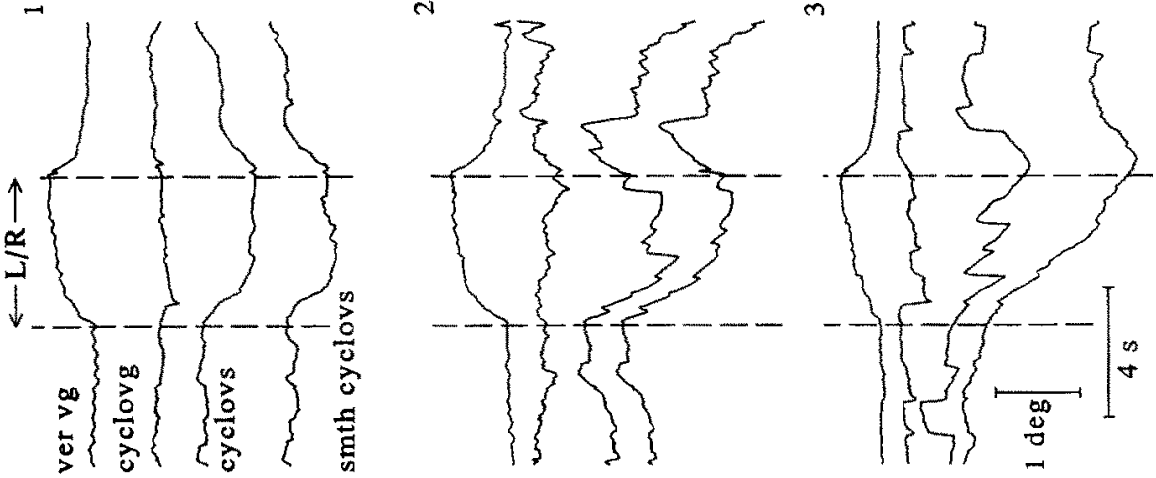

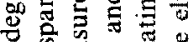

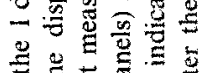

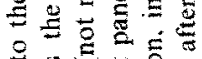

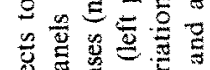

送言赑

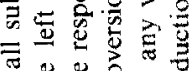

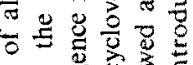

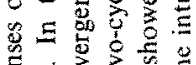

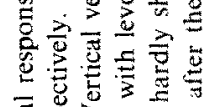

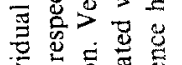

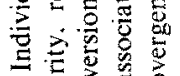

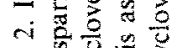

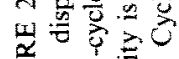

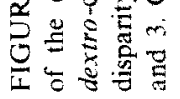



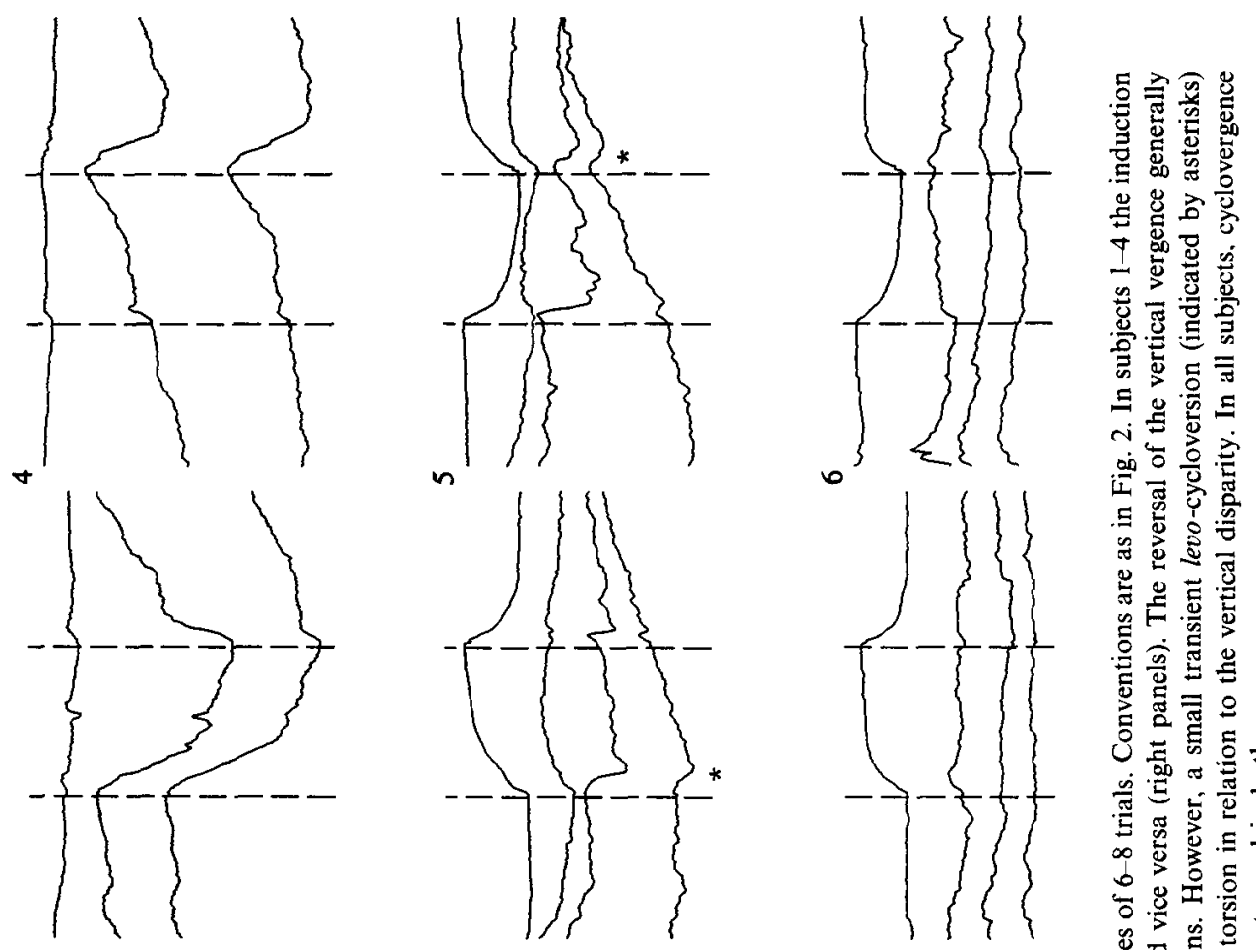

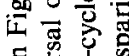

क :

莺总总

家焉焉

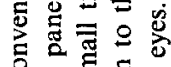

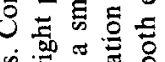

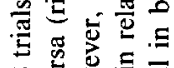

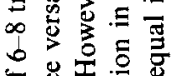

은

总总

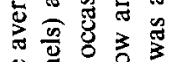
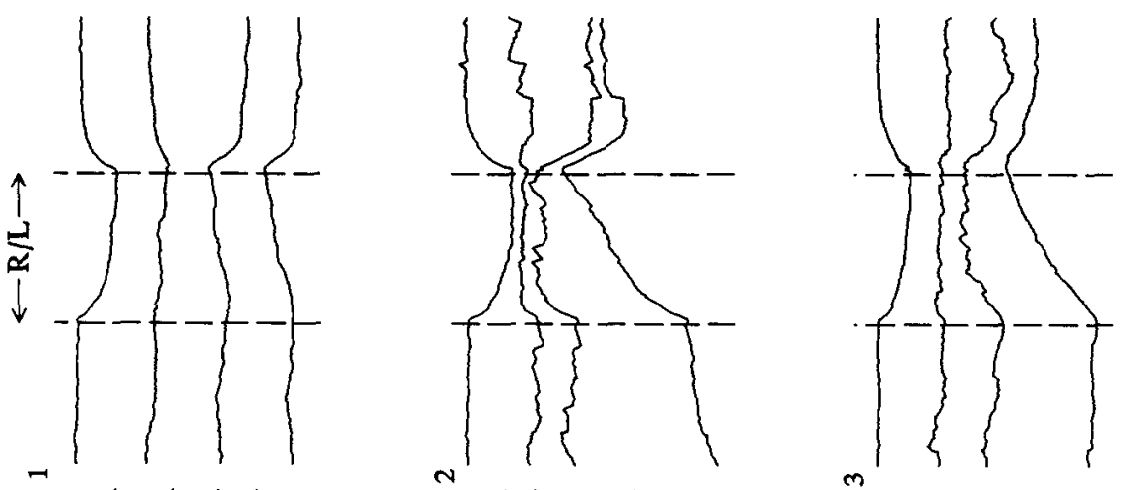

焉

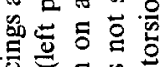

昰

的可

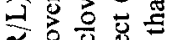

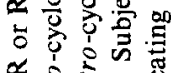

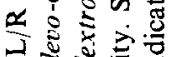

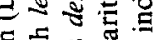

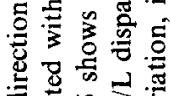

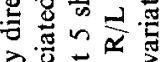
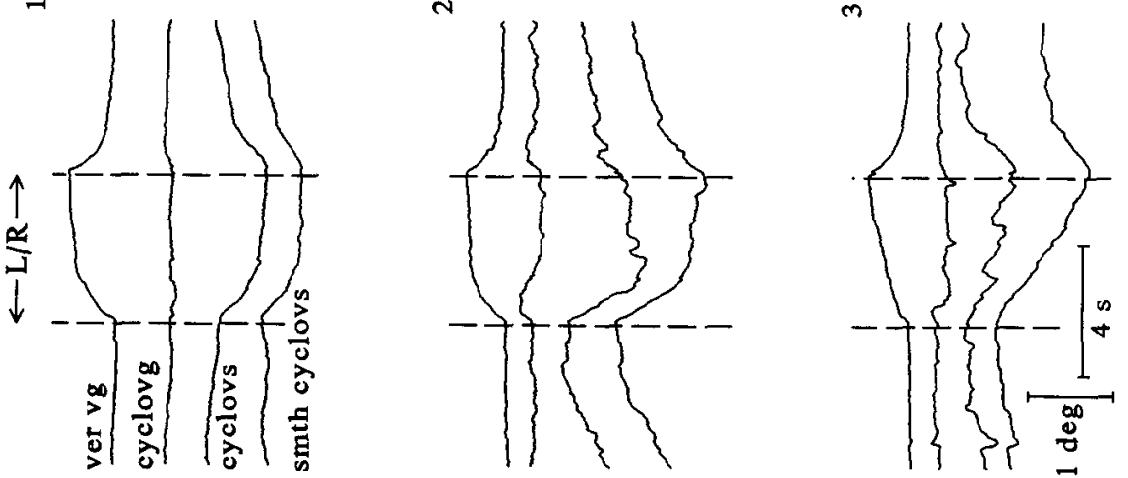

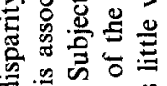

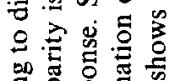

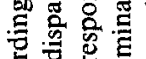

造总部

恶密品

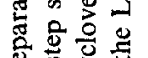

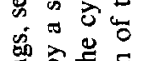

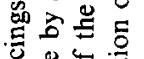

递它范

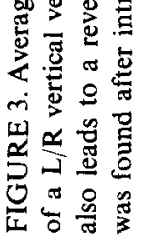


displayed a dextro-cycloversional nystagmus, independent of the direction of the vertical disparity step (see Figs 2 and 3, fifth panels). Only during a very short time interval after a $L / R$ disparity step (or after the reversal of a $\mathrm{R} / \mathrm{L}$ disparity step) did this subject display a nystagmus with the slow phases in levo-cycloversional direction (indicated by asterisks in Fig. 3). The sixth subject failed to show any torsion in association with vertical vergence (Fig. 2 and 3, sixth panels).

Disparity elimination. In subjects 1-4, the elimination of the stimulus disparity led to a reversal of the cycloversion, independent of whether the disparity was $L / R$ (left panels) or R/L (right panels). In subjects 2 and 4 this reversal was only transient. This may be caused by the rapid takeover of the "normal" cycloversional nystagmus that was observed prior to disparity introduction in these two subjects (see above). Subject 5 continued to show a dextro-cycloversional nystagmus, independent of the direction of the preceding vertical disparity. Only after elimination of a right-over-left disparity the cycloversion was, transiently, in levo-cycloversional direction. As was the case for disparity introduction, in subject 6 the elimination of the disparity did not elicit eye torsion.

Association with horizontal and vertical version. The cycloversional nystagmus movements that were described above did not occur, altogether, in isolation. Cycloversional fast phases were accompanied by microsaccades in horizontal and vertical direction. Subjects 2 and 3 displayed a small vertical nystagmus that was upbeat and downbeat in these two subjects, respectively. The fast phases of this vertical nystagmus coincided with the fast phases of the cycloversional nystagmus and the amplitudes of the vertical and cycloversional nystagmus were in the same order of magnitude. Subject 6 also displayed a small vertical (upbeat) nystagmus, although in this subject there was no cycloversional nystagmus. Notably, in subjects 2, 3 and 6 this vertical nystagmus was also present in the (calibration) measurements in which no vertical disparity was presented.

Head fixation in subject 6 . The absence of any cycloversion in association with vertical vergence in one subject (subject 6) is a strong argument against the superior oblique hypothesis of Enright (see Discussion). In theory, however, this absence may be caused by compensatory torsional head movements. In order to exclude this latter possibility, we repeated the experiment in subject 6 , but fixated the subject's head by a biteboard. The results were identical: neither the introduction of the disparity nor its elimination elicited cycloversion.

\section{Role of gaze direction}

In abduction the superior oblique muscle will act more as a cyclorotator than in adduction. Therefore, if one postulates (as Enright) that vertical vergence is mediated

*The advantage of using cyclovergence rather than torsion of the individual eyes is that the former is less contaminated by spontaneous variation. See Van Rijn et al. (1994). predominantly by the superior oblique muscles, one should expect that an abducted eve will cyclorotate more than an adducted eye.

We tested this prediction in subject 2, who showed the vertical vergence-cycloversion association very markedly. We presented to this subject the stimulus at various azimuth angles: $20 \mathrm{deg}$ left, straight ahead and $20 \mathrm{deg}$ right. One should expect $e x$-cyclovergence when looking at a $\mathrm{L} / \mathrm{R}$ disparity that is presented rightward with respect to the straight ahead. A $\mathrm{R} / \mathrm{L}$ disparity should induce in-cyclovergence when presented in this direction. Disparities presented leftward from the straight ahead should induce cyclovergence with the opposite sign.

If the superior oblique muscle would act as a string attached to the globe, the ratio between torsion and elevation should equal the sine of the insertion angle. Since in straight ahead direction the insertion angle equals about $51 \mathrm{deg}$ (Von Noorden, 1990), one may calculate that in $20 \mathrm{deg}$ lateral gaze, cyclovergence should be about $60 \%$ of cycloversion.* The pulling direction may rotate, to some extent, along with rotations of the globe [the "no-pully" model proposed by Miller and Robbins (1987) (see Miller, 1989)]. Therefore the torsion/elevation ratio may be less dependent on the insertion angle and cyclovergence may be considerably smaller. However, it should still be in the indicated direction.

The results from our experiment, shown in Fig. 4 (avcraged responses), demonstrate that cyclovergence varied hardly at all. Moreover, cyclovergence did not systematically depend on either the horizontal gaze direction or the direction of the vertical disparity. In contrast, cycloversion was elicited in all three horizontal gaze directions. in a way that was similar to that found in the first experiment.

\section{DISCUSSION}

A primary purpose of these experiments was to try to reproduce the results of Enright (1992), who found that disparity-induced vertical vergence is associated with cycloversion. We largely confirmed his results, but found that the response was partly in the form of a cycloversional nystagmus rather than a tonic response. In a specific experiment we tested and rejected the hypothesis offered by Enright to explain his findings. Furthermore, our present results may relate to the spontaneous torsional nystagmus that was observed in this and earlier experiments. In the final section of this discussion we will compare the characteristics of our vertical vergence responses to findings reported in the litcrature.

\section{Vertical vergence es cycloversion}

In the present experiments we confirm, in four of our six subjects, the findings of Enright that cycloversion is coupled to vertical vergence. However, we found that vertical vergence partly induced a cycloversional nystagmus rather than an exclusively tonic cycloversion. This cycloversion was rightward for $\mathrm{R} / \mathrm{L}$ vertical disparities 


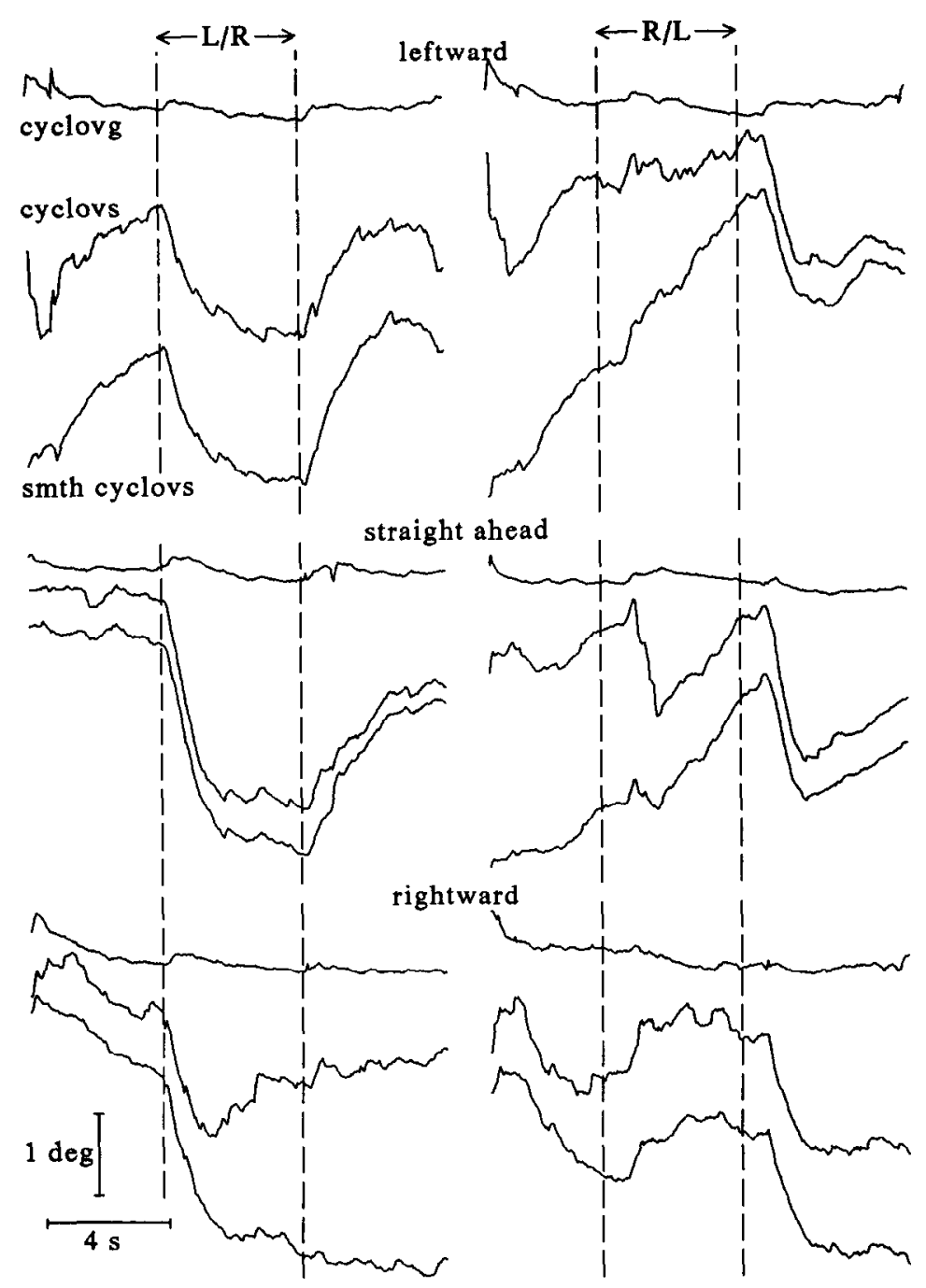

FIGURE 4. Cycloversion and cyclovergence responses in different horizontal gaze directions (subject 2): "leftward" and "rightward" indicate gaze angles that are rotated $20 \mathrm{deg}$ with respect to the straight ahead. Tracings are averages of 3-6 trials. Conventions are as in Fig. 2. Cyclovergence does not systematically depend on either horizontal gaze angle or the direction of the disparity. Although cycloversion responses were variable, the pattern largely corresponds to that found in the earlier experiment (see Figs 2 and 3). The transicnt cx-cyclovergence at the beginning of most tracings is an aftereffect of blinking during inter-trial intervals. Due to technical problems, vertical vergence was not recorded in this experiment.

and leftward for $\mathrm{L} / \mathrm{R}$ disparities. The slow phase velocity of the cycloversional nystagmus cither decrcased exponentially or was constant, depending on subject and disparity direction.

Enright found that the cycloversion responses were about $40 \%$ of the vertical disparity size (which was $0.86 \mathrm{deg}$ ). We found the size of the tonic components of the cycloversion responses to be about equal to that of the vertical vergence (Fig. 2, saccades present). If we take into consideration that the gain of the vertical vergence was about 0.55 , our response magnitudes are in agreement with those of Enright.

\section{Mechanism}

The hypothesis offered by Enright to explain his findings is that vertical vergence is mediated predominantly by the superior oblique muscles. Contraction of this muscle produces eye depression and also intorsion. Relaxation produces the opposite effects. Enright found support for this hypothesis in his findings that vertical vergence was associated with a slight lateral displacement of the eye, in a direction that was in agreement with expected contraction or relaxation of the superior oblique muscle.

Our findings are in conflict with this hypothesis in three ways. Firstly, according to this hypothesis one should anticipate a tonic cycloversion rather than a cycloversional nystagmus since the tonic vertical vergence requires a tonic contraction of the superior oblique muscle. The fact that in our experiment the cycloversion was often nystagmic in nature strongly suggests that the origin is central and not peripheral. Secondly, a mechanical coupling, as proposed by Enright, predicts a firm relation between vertical vergence and cycloversion in all subjects. However, we find this relation to be idiosyncratic: in subject 5 we only found dextrocycloversion, independent of the direction of the vertical vergence; in subject 6 there was no cycloversion at all. In a repeated experiment in subject 6 we fixated the head by a biteboard and demonstrated that this lack of cycloversion 
was not caused by compensatory torsional head movements (i.e. eye-in-head torsion and head-in-space torsion adding to zero eye-in-space torsion). Thirdly, Enright's hypothesis leads to the prediction that the relative contribution of the left and right eyes to the cycloversion response should depend on the horizontal gaze angle. Hence, one should expect that cyclovergence shows typical changes depending on the gaze direction in which the vertical vergence is elicited. Contrary to this prediction, our second experiment in subject 2 (Fig. 4) demonstrates that cyclovergence does not depend on either horizontal gaze direction or direction of vertical disparity. In our view this second argument may be weakened but certainly not overthrown by the uncertainty about the exact relation between torsion and elevation in various horizontal gaze angles [no-pully model of ocular muscles (see Miller. 1989)].

Another association between vertical vergence and cycloversion exists in the so-called ocular tilt reaction. In this reaction, head tilt, vertical vergence and cycloversion are elicited by stimulation of various brain stem parts. It was first described by Westheimer and Blair (1975), who stimulated, in a monkey, a brain stem area that included the interstitial nucleus of Cajal (INC). Later investigators reported similar associations between vertical vergence and cycloversion in a human patient after stimulation of this nucleus (Lueck, Hamlyn, Crawford, Levy, Brindley, Watkins \& Kennard, 1991) and in patients with central (Lopez, Bronstein, Gresty, Rudge \& Du Boulay, 1992) and peripheral (Halmagyi, Gresty \& Gibson. 1979) vestibular lesions. In all these reports. the direction of the association is reversed with respect to our results. In the ocular tilt reaction, left over right vertical vergence is associated with dextro-cycloversion, i.e. a rotation of the upper poles towards the right shoulder. It has been suggested that the ocular tilt reaction may be related to the vestibulo-ocular reflex for roll head movements in animals with lateral eyes (Lopez et al., 1992). Because of the reversed association it is unlikely that the relation between disparity induced vertical vergence and cycloversion that we report is an epiphenomenon of this ocular tilt reaction.

Despite the questions on the physiological meaning of the association the occurrence of a cycloversional nystagmus does strongly suggest a central rather than peripheral origin. Vertical and torsional eye movements are mediated by the same promotor nuclei in the brain stem: the rostral interstitial nucleus of the medial longitudinal fasciculus (riMLF) mediates fast eye movements and the INC serves as the neural integrator for these movement types. Independently of the physiological role, the observed association may have its origin in some coupling at the level of these premotor nuclei.

Association of cycloversion with horizontal and vertical version

We found that the fast phases of the cycloversional nystagmus in our subjects coincided with horizontal and/or vertical microsaccades or with the fast phases of a small vertical nystagmus. This vertical nystagmus appeared not to be related to the presentation of the vertical disparities since it was present in the calibration measurements as well. Both the occurrence of spontaneous microsaccades and the small vertical nystagmus have been documented in the literature [microsaccades (see e.g. Steinman, Haddad, Skavenski \& Wyman, 1973 ; Ditchburn. 1980; Kowler \& Steinman. 1980); vertical nystagmus (see Van Rijn el al., 1994)]. Our results demonstrate that the cycloversional nystagmus is, in a way, superimposed on these movements in horizontal and vertical directions. The fact that cycloversional fast phases coincide with these horizontal and vertical saccades does not corroborate our findings with respect to the association between vertical vergence and torsion. nor does it disqualify our hypotheses with respect to its origin. It merely demonstrates that saccadic control occurs for the three movement dimensions simultaneously (see e.g. Crawford \& Vilis, 1991). A discussion of this issue falls, however, outside the realm of this article.

\section{Relation to vertical phoria}

In subjects 2 and 4 we observed a systematic drift of spontaneous cycloversion. In subject 5 there was a preference for dextrocycloversion after introduction as well as elimination of the vertical disparity. The relation between vertical vergence and cycloversion that we found in the present experiment gives rise to the hypothesis that this directional preponderance of cycloversion may be caused by a vertical phoria that requires continuous vertical disparity vergence. This might also explain the spontaneous torsional (cycloversional) nystagmus that we observed in a previous experiment (Van Rijn et al., 1994). This torsional nystagmus was enhanced by a rich visual background. This may fit in this hypothesis since a rich background is more compelling for fusion and therefore also for disparity vergence.

In order to get an indication of the answer to this question we compared vertical phorias in our subjects with the directional preponderance of the cycloversion as estimated from Figs 2 and 3. Results are listed in Table 1. The vertical phorias, when present, were $L / R$ and preponderances of cycloversion, when present, were in

TABLE 1. Vertical phorias and cycloversional directional preponderances in our subjects

\begin{tabular}{lcc}
\hline Subject & Vertical phoria & $\begin{array}{c}\text { Cycloversional (slow phase) } \\
\text { directional preponderance }\end{array}$ \\
\hline 1 & 0.0 & None \\
2 & $0.2 \mathrm{~L} / \mathrm{R}$ & Dextro-cycloversion \\
3 & $0.2 \mathrm{~L} / \mathrm{R}$ & None \\
4 & 0.0 & Dextro-cycloversion \\
5 & $0.6 \mathrm{~L} / \mathrm{R}$ & Dextro-cycloversion \\
6 & $0.3 \mathrm{~L} / \mathrm{R}$ & None \\
\hline
\end{tabular}

Vertical phoria values were obtained with a Maddox cross at $1.45 \mathrm{~m}$ distance (coinciding with the distance of the stimulus during the experiment) and represent averages of three measurements. If values were different for left and right eye coverage, the maximum of these values is listed. The directional preponderances of cycloversion are estimated from Fig. 3 
dextro-cycloversional direction. Since a $L / R$ phoria requires a $R / L$ vertical vergence (in absence of disparity), the direction of the association between cycloversion and vertical vergence is similar to that found during disparity presentations. At first sight this seems to support a relation between vertical phoria and cycloversional direction preponderance.

A complicating factor is that disparity vergence appears to have a fast and a tonic component (Ellerbrock, 1950; Ludvigh, Mckinnon \& Zaitzeff, 1964; Carter, 1965; Schor, 1979; Seth \& Henson, 1984). The fast component may be stimulated directly by the disparity whereas the slow component may be affected by the duration of the fast vergence presence. These fast and slow components may be distinguished by the time course of their relaxation. The slow component, which predominates when the disparity is presented during a longer time interval, may persist during several hours after monocular occlusion (Alpern, 1946). It seems possible that these fast and slow components of vertical disparity vergence are related differently to cycloversion. The fast component of vertical vergence most likely has a direct relation to cycloversion. This may be observed in Figs 2 and 3 . Upon elimination of the vertical disparity there is, in all five subjects that show torsion responses, at least a temporary reversal of the cycloversion response along with the reversal of vertical vergence. It must be noted that disparity elimination (Figs 2 and 3 ) is not identical to vergence relaxation (Schor, 1979) since in the former case a zero disparity is presented and in the latter one eye is covered. However, since the presentation time of the disparity was short (only $4 \mathrm{sec}$ ) it seems unlikely that the slow vergence system is involved to a large extent.

In the correction of vertical phoria the slow vergence system is much more important than the fast system since, obviously, the duration of the disparity presentation is much longer. The question therefore is whether slow vertical vergence is also associated with cycloversion. This question cannot be directly answered from our data since all our measurements were short term.

\section{Vertical vergence}

These results will be discussed only briefly since a study of the vertical vergence system was not the main purpose of this experiment. We found that a step vertical disparity induced a vertical vergence response with a gain of about 0.55 and a time constant of about $0.94 \mathrm{sec}$ (on establishment of the disparity). In general, subjects obtained a single, fused image. Kertesz (1981), investigating the maximum disparity that could be fused, found gain values of 0.63 and 0.75 in his two subjects. Our value seems low in relation to those of Kertesz, but we note that Kertesz used randomly segmented horizontal lines as a stimulus, which may be more compelling for vertical vergence than our concentric circles. The rationale behind our stimulus choice has been explained in the Method section. We note that Perlmutter and Kertesz (1978) found gain values between $0.80 \%$ and $1.00 \%$ for the vertical vergence response to a step disparity of $1 \mathrm{deg}$ in a single horizontal line. This result seems in disagreement with ours, although we cannot exclude the possibility that even a single horizontal line is more compelling for vertical vergence than our stimulus.

For the return movement (after disparity elimination) we found a time constant of about $0.60 \mathrm{sec}$. In the literature, much longer values have been reported. Houtman, Roze and Scheper (1977) found time constants of between 3 and $5 \mathrm{sec}$. Henson and North (1980) and Ellerbrock (1950) found values of about 1 and several min, respectively, as estimated from their figures. In all these investigations, disparity presentation times were much longer than in ours, which may explain, though differences in tonic parts of the responses, the differences in the time constants.

\section{REFERENCES}

Alpern, M. A. (1946). The after effect of lateral duction testing on subsequent phoria measurements. American Journal of Optometry, 23, $442-447$.

Carpenter, R. H. S. (1988). Movements of the eyes. ( 2 nd edn). London: Pion.

Carter, D. B. (1965). Fixation disparity and heterophoria following prolonged wearing of prisms. American Journal of Optometry, 42, $141-152$.

Crawford, J. D. \& Vilis. T. (1991). Axes of rotation and Listing's law during rotations of the head. Journal of Neurophysiology, 65, $407-423$.

Ditchburn, R. W. (1980). The function of small saccades. Vision Research, 20, 271-272.

Ellerbrock, V. J. (1950). Tonicity induced by fusional movements. American Journal of Optometry, 27, 8-20

Enright, J. T. (1992). Unexpected role of the oblique muscles in the human vertical fusional reflex. Journal of Physiology, 451 . 279-293.

Ferman, L., Collewijn, H., Jansen. T. C. \& Van den Berg, A. V. (1987). Human gaze stability in the horizontal, vertical and torsional direction during voluntary head movements, evaluated with a three-dimensional scleral induction coil technique. Vision Research, 27, $811-828$.

Halmagyi, G. M., Gresty, M. A. \& Gibson, W. P. R. (1979). Ocular tilt reaction with peripheral vestibular lesion. Annals of Neurology, $6,80-83$.

Henson, D. B. \& North. R. (1980). Adaptation to prism induced heterophoria. American Journal of Optometry and Physiological Optics, 57, 129-137.

Houtman, W. A., Roze, J. H. \& Scheper, W. (1977), Vertical motor fusion. Documenta Ophthalmologica, 44, 179-185.

Howard, I. P. \& Zacher, I. E. (1991). Human cyclovergence as a function of stimulus frequency and amplitude. Experimental Brain Research, 85, 445-450.

Kertesz, A. E. (1981). Effect of stimulus size on fusion and vergence. Journal of the Optical Society of America, 71, 289-293.

Kertesz, A. E. (1983). Vertical and cyclofusional disparity vergence. In Schor, C. M. \& Ciuffreda, K. J. (Eds), Vergence eye movements: Basic and clinical aspects (pp. 317-348). London: Butterworths.

Kowler, E. \& Steinman, R. M. (1980). Small saccades serve no useful purpose: Reply to a letter by R. W. Ditchburn. Vision Research, 20, 273-276

Lopez, L., Bronstein, A. M., Gresty, M. A., Rudge, P. \& Du Boulay, E. P. G. H. (1992). Torsional nystagmus. A neuro-otological and MRI study of thirty-five cases. Brain, 115, 1107-1124.

Ludvigh, E., Mckinnon, P. \& Zaitzeff, L. (1964). Temporal course of the relaxation of binocular duction (fusion) movements. Archives of Ophthalmology, 71, 389-399.

Lueck, C. J., Hamlyn, P., Crawford, T. J., Levy, I. S., Brindley, G. S., Watkins, E. S. \& Kennard, C. (1991). A case of ocular tilt reaction 
and torsional nystagmus due to direct stimulation of the midbrain in man. Brain, 1/4, 2069-2079.

Miller, J. M. (1989). Functional anatomy of normal human rectus muscles. Vision Research, 29, 223-240.

Miller, J. M. \& Robbins, D. (1987). Extraocular muscle sideslip and orbital geometry in monkeys. Vision Research, 27, 381-392

Perlmutter, A. L. \& Kertesz, A. E. (1978). Measurement of human vertical fusional response. Vision Research, 18, 219-223.

Robinson. D. A. (1963). A method of measuring eye movement using a scleral search coil in a magnetic field. IEEE Transactions on Biomedical Electronics, BME-10, 137-145.

Schor, C. M. (1979). The relationship between fusional vergence eye movements and fixation disparity. Vision Research, 19, 13591367.

Sethi, B. \& Henson, D. B. (1984). Adaptive changes with prolonged effects of comitant and incomitant vergence disparities. American Journal of Optometry and Physiological Optics, 61, 506-512.

Steinman, R. M., Haddad, G. M., Skavenski, A. A. \& Wyman. D. (1973). Miniature eye movements. Science. 181. 810-818.
Van Riin, L. J. \& Van den Berg, A. V. (1993). Binocular eye orientation during fixations: Listing's law extended to include eye vergence. Vision Rescarch, 33.691 708.

Van Rijn, L. J., Van der Steen, J. \& Collewijn, H. (1992). Visuallyinduced cycloversion and cyclovergence. Vision Research. 32, 1875- 1883.

Van Rijn, L. I., Van der Steen, J. \& Collewijn, H. (1994). Instability of ocular torsion during fixation: Cyclovergence is more stable than cycloversion. Vision Research, 34, 1077-1087.

Von Noorden, G. K. (1990), Von Noorden-Burian's binocular cision and ocular mobility. Theory and management of strabismus (4th edn). St Louis, Mo. Mosby.

Westheimer, G. \& Blair, S. M. (1975). The ocular tilt reaction-a brainstem oculomotor routine. Investigative Ophthalmology and Visual Science, 14.833-839.

Acknowledgements - We thank Dr J. T. Enright for his critical review of the manuscript and for his valuable suggestion which contributed to the logic of this paper. 